\title{
GRAVIDEZ NA ADOLESCÊNCIA: ANÁLISE CONTEXTUAL DE RISCO E PROTEÇÃO
}

\author{
Elder Cerqueira-Santos \\ Simone dos Santos Paludo \\ Eva Diniz Bensaja dei Schirò \\ Sílvia Helena Koller
}

\begin{abstract}
RESUMO. A gravidez precoce pode ser entendida como fator de risco ou proteção no adolescente. É crescente a necessidade de pesquisas que aprofundem os aspectos envolvidos nessa questão. Este estudo exploratório-descritivo teve como objetivo identificar as diferenças existentes entre um grupo de adolescentes que viveu a experiência de gravidez durante a adolescência. A amostra foi composta por 1015 jovens de nível socioeconômico baixo de Porto Alegre, com 14-24 anos, de ambos os sexos. Utilizou-se um questionário com 109 questões que abordam aspectos da vida juvenil: drogas, sexualidade, religiosidade, escola. Observou-se maior número de participantes do sexo feminino $52.3 \%$ contra $47.7 \%$ do sexo masculino. $53.5 \%$ dos jovens já iniciou a vida sexual, dentre estes, $64.6 \%$ eram homens. A idade média para a primeira relação foi significativamente menor para eles $(M=13.64$ anos; $d p=1.7)$ do que para elas $(M=14.79$ anos; $d p=1.4)$. Os resultados revelam um percentual esperado para gravidez e aborto em jovens de nível socioeconômico baixo, quando comparado a outros estudos. Sobressai a baixa idade para a primeira relação sexual e o fato de que cerca de $1 / 4$ da amostra apresentar uso irregular de métodos contraceptivos. Discute-se sobre uso de métodos anticoncepcionais e aborto, associadas à saúde do adolescente.

Palavras-chave: Gravidez; adolescência; risco e proteção.
\end{abstract}

\section{EARLY PREGNANCY: A CONTEXTUAL ANALYSIS ABOUT RISK AND PROTECTION}

\begin{abstract}
Early pregnancy has been widely understood as a risk factor, moreover, in some cases, it has been considering as a protective factor. Thus, more studies among this population should be done, looking for the variables involving such question. This exploratory-descriptive study had as aim to identify the existent differences among a group of adolescents that lived the pregnancy experience. Participants were 1015 low SES young people from Porto Alegre, aged between 14-24, males and females. A questionnaire with 109 questions was used asking about major domains of life: drug use, sexuality, religiosity, school. There were more females $52.3 \%$ than males $47.7 \%$ in the sample. Most of the participants already had their first sexual intercourse, 53.5\%, most of them males $(64.6 \%)$. The average for first sexual intercourse was lower for males $(M=13.64 ; d p=1.7)$ than females $(M=14.79 ; d p=1.4)$. Results indicate an expected rate of pregnancy and abortion for low SES young. We stress the low age for first sexual intercourse and the fact that $1 / 4$ of our sample has an irregular contraceptive method. We discuss about contraceptive method and abortion linked to the adolescents health.
\end{abstract}

Key words: Pregnancy; adolescence; risk and protection.

\section{GRAVIDEZ EN LA ADOLESCENCIA: ANÁLISIS CONTEXTUAL DEL RIESGO E PROTECCIÓN}

\footnotetext{
Doutor em Psicologia pela UFRGS. Professor da Universidade Federal de Sergipe (UFS).

\# Doutora em Psicologia. Professora da Universidade do Rio Grande (FURG).

ฯ Doutoranda em Psicologia pela Universidade Federal de Rio Grande do Sul (UFRGS).

æ Doutora em Educação. Professora Associada II da Universidade Federal do Rio Grande do Sul.
}

RESUMEN. El embarazo precoz puede ser entendida como un factor de riesgo o protección para el adolescente. La necesidad de investigaciones que profundicen los aspectos involucrados en esta cuestión es creciente. Hecho en Porto Alegre, capital de la provincia de Rio Grande do Sul, ubicada al sur de Brasil, este es un estudio exploratorio descriptivo, con una muestra de 1015 jóvenes de ambos sexos con edad entre 14 y 24 años y de bajo nivel socio económico. La investigación se valió de una encuesta con 109 cuestiones que abordan aspectos de la vida juvenil: drogas, sexualidad, religiosidad, escuela. El porcentaje de los encuestados correspondiente al sexo femenino fue de $52.3 \%$ y $47.7 \%$ del sexo masculino. De estos jóvenes $53.5 \%$ ya 
empezó la vida sexual, entre los cuales, $64.6 \%$ eran hombres. De hecho, de la observación de los datos se desprende una clara tendencia a una mayor precocidad para los hombres $(M=13.64$ anos; $d p=1.7)$ aunque la media de edad de las mujeres para iniciarse en las relaciones sexuales no esté muy por debajo $(M=14.79$ anos; $d p=1.4)$. El análisis de los datos revela un porcentual esperado para embarazo y aborto en jóvenes de bajo nivel socio económico, lo que corrobora los resultados de otros estudios ya publicados. Sobresale en la encuesta la edad precoz para el primer encuentro sexual y el hecho de que al rededor de $1 / 4$ de la muestra hace uso irregular de los métodos contraceptivos. Plantease sobre el uso de los métodos anticonceptivos y sobre el aborto, asociados a la salud del adolescente.

Palabras-clave: Embarazo; adolescencia; riesco y protección.

\begin{abstract}
A minha mãe disse que me teve quando ela tinha 16 anos. Naquela época não era muito cedo não. Tava bom! Fazer o que? Mas hoje eu não quero isso pra mim. O tempo mudou. Tem que estudar mais, um filho ia acabar com a minha vida! Eu nem conto pra todo mundo que já fiquei grávida. (Paula, 17 anos, estudante, uma gestação interrompida aos 16 anos).
\end{abstract}

Dados sobre a gravidez na adolescência vêm mostrando um aumento na taxa de fecundidade para esta população quando comparada a mulheres adultas, especialmente nos países mais pobres, como é o caso da América Latina. Segundo o Estatuto da Criança e do Adolescente, Lei n. ${ }^{\circ}$ 8069/90, adolescente é todo indivíduo com idade entre 12 e 18 anos e para a Organização Mundial de Saúde (OMS) esse período envolve indivíduos com idades entre 10 a 19 anos. Além das mudanças físicas impostas pela faixa etária, a adolescência envolve um período de profundas mudanças biopsicossociais, especialmente relacionadas à maturação sexual, a busca da identidade adulta e a autonomização frente aos pais. A gravidez nesse momento de vida oferece implicações desenvolvimentais tanto para o adolescente quanto para aqueles envolvidos nessa situação. A literatura tem tratado a gravidez na adolescência como um problema de saúde pública, especialmente pelo fato de propiciar riscos ao desenvolvimento da criança gerada e da própria adolescente gestante (Gontijo \& Medeiros, 2004). No entanto, nem sempre a repercussão da gravidez pode ser identificada como um fator de risco. Cowan, Cowan e Shulz (1996) salientaram que os fatores de risco relacionam-se com eventos negativos de vida que, quando presentes, aumentam a probabilidade da pessoa apresentar problemas, mas reiteram que o risco deve ser visto como um processo e não uma única variável. Tal fato permite uma problematização do fenômeno da gravidez como risco e/ou proteção. Ao contextualizar a gravidez para aqueles adolescentes que vivem em situação de pobreza, o presente estudo buscou identificar os fatores de risco e os fatores de proteção associados a este acontecimento.

No Brasil, estima-se que aproximadamente 20$25 \%$ do total de mulheres gestantes são adolescentes, apontando que uma em cada cinco gestantes são adolescentes entre 14 e 20 anos de idade (Santos Júnior, 1999). Além disso, verifica-se que no Brasil, se assiste a um aumento do número de adolescentes que engravidam. Ao contrário do que acontece nos restantes países ocidentais, nos quais tende a ocorrer uma diminuição na ocorrência deste evento (Pesquisa GRAVAD, 2006).

Em levantamento realizado em 2004, Szwarcwald, Júnior, Pascom e Júnior (2004) constataram que os adolescentes brasileiros têm iniciado a vida sexual mais cedo e mantêm um maior número de parceiros. Segundo o Ministério da Saúde (2006), 36\% dos jovens entre 15-24 anos relataram ter tido a primeira relação sexual antes dos 15 anos de idade, enquanto apenas $21 \%$ dos jovens entre 25-29 anos tiveram a primeira relação na mesma época. Destes, 20\% afirmaram ter tido mais de dez parceiros nas suas vidas e $7 \%$ tiveram mais de cinco parceiros no último ano.

O aumento nas taxas de gravidez na adolescência pode ser explicado por diferentes causas, podendo variar de país para país. Dentre a complexidade de fatores de risco para analisar esta questão, destacam-se os aspectos socioeconômicos. Apesar do fenômeno atingir e estar crescente em todas as classes sociais, ainda há uma forte relação entre pobreza, baixa escolaridade e a baixa idade para gravidez. Além disso, fatores como a diminuição global para a idade média para menarca e da primeira relação sexual compõem um cenário de risco que colabora para o aumento dessas taxas. O estudo de Moura (1991), mostrou que no estado de São Paulo, a idade média para a menarca diminuiu significativamente de 13 para 11 anos de idade em uma década. De forma semelhante, o estudo de Cerqueira-Santos (2007), realizado em quatro capitais brasileiras, apontou que a idade média de iniciação sexual dos jovens de nível 
socioeconômico baixo está por volta dos 13 anos. Estudos anteriores, da década de 90, revisados por Santos Júnior (1999), revelavam médias entre 15 e 17 anos para a primeira relação sexual desta população. Aquino et. al. (2003), em estudo multicêntrico no Brasil, encontraram que a prevalência de gravidez antes dos 18 anos de idade (maioridade legal brasileira) foi relatada por $8,9 \%$ dos homens e $16,6 \%$ das mulheres. O mesmo estudo relatou que a maior parte dos episódios de gravidez para esta população aconteceu no contexto de um relacionamento afetivo, sendo maior o relato masculino sobre a gravidez de uma parceira eventual do que um relato feminino sobre esta situação. Destacou-se, ainda, neste estudo o fato de que a ocorrência de uma gravidez antes dos vinte anos variou inversamente com a renda e a escolaridade.

Dados do Ministério da Saúde (2002) também indicaram que, nessa faixa etária (adolescência), a proporção de gravidez é de $23,5 \%$. Nas meninas com idade inferior a 15 anos, este valor é de $0,9 \%$ e para aquelas entre os 15 e os 19 anos, 22,6\%. Estes percentuais apresentam variações nos diferentes estados brasileiros, observando-se que São Paulo é aquele que apresenta uma incidência mais reduzida (19,5\%), enquanto no Maranhão e Tocantins estes valores tomam uma maior dominância $(32,3 \%)$ (Ministério da Saúde, 2006; Aquino et al., 2003).

Em 2006, num relatório do Ministério da Saúde, observou-se que a taxa específica de fecundidade (número de filhos, por faixa etária e região/população total dessa faixa etária nessa região) para adolescentes, com idades compreendidas entre os 15-19 anos era de 0,0714 no Brasil. Sendo que a maior incidência deste fenômeno ocorre no Norte $(0,1034)$ do país, seguida do Nordeste $(0,0860)$, Centro-Oeste $(0,0818)$, Sul $(0,0579)$ e Sudeste $(0,0561)$.Observa-se que a gravidez na adolescência tem diferentes causas e envolve diferentes fatores de risco como, por exemplo, o crescimento da população de jovens e as modificações na forma como é atualmente vivida a sexualidade (Lima et al., 2004). Neste contexto, discute-se o conhecimento e o uso de métodos contraceptivos de diferentes maneiras. Algumas pesquisas apontam como risco para a gravidez na adolescência o início da vida sexual, aliada à falta de informação sobre meios contraceptivos e à deficiência de programas de apoio ao adolescente (Sabroza, et al., 2004). Tradicionalmente, a gravidez na adolescência era descrita como um problema social, associada à pobreza, encarada como comprometedora de um desenvolvimento saudável, tanto para a mãe, como para o seu filho. Contudo, estudos mais recentes descrevem o fenômeno de forma distinta, como resultante de múltiplas características e variáveis influenciadoras do desenvolvimento (Canavarro \& Pereira, 2001). A maternidade adolescente é descrita como um produto de vários fatores de risco, nomeadamente, a história desenvolvimental dos pais, nível socioeconômico, redes de apoio, recursos psicológicos, idade dos progenitores a características de temperamento e desenvolvimentais do bebê (Jacard, Dodge, \& Dittus, 2003).

Nesta perspectiva, é considerado redutor descrever a gravidez adolescente como um grupo homogêneo de risco, já que este é um fenômeno que ocorre numa variedade de transações possíveis e a vulnerabilidade de um dos elementos (por exemplo, mãe/bebê) poderá ser minimizado pela potencialidade de outros que, poderão funcionar como fatores protetores. De acordo com Rutter $(1985,1987)$ os fatores de proteção são compreendidos como aqueles que modificam, melhoram ou alteram as respostas pessoais a determinados riscos de desadaptação. Os atributos disposicionais das pessoas, a rede de apoio social e a coesão familiar são fatores protetivos que quando presentes contribuem para o enfrentamento do risco (Masten \& Garmezy, 1985). Na vivência da gravidez na adolescência quanto maior o número de recursos internos e externos, maior a possibilidade de sucesso da unidade familiar, o risco poderá ser maximizado ou minimizado perante outras variáveis (Figueiredo, 2000).

Como lembram Heilborn et al. (2002), sob o termo "gravidez na adolescência" encontra-se uma faixa etária para a qual, por muito tempo, foi a época da vida ideal para ter um filho. Para Heilborn et al. (2002) o fenômeno da gravidez na adolescência "também ganha importância no cenário de mudanças operadas na concepção social das idades e do sexo que redefinem as expectativas sociais depositadas nos jovens nos dias atuais, sobretudo nas adolescentes do sexo feminino" (p.18). Os autores continuam argumentando que diante desta possibilidade atual de vivência da sexualidade desvinculada da reprodução, a gravidez se coloca como uma perda de oportunidades de vivências na juventude. Por esse motivo, a gravidez adolescente tende a ser indicada como um fator de risco no desenvolvimento, tanto dos pais como da criança, uma vez que se constitui um desafio para aqueles nela envolvidos (Canavarro \& Pereira, 2001; Levandowski \& Piccinini, 2004; Soares, Marques, Martins, Figueiredo, Jongenelen, \& Matos, 2002).

A perspectiva de que a gravidez deverá surgir num contexto de planejamento, estabilidade econômica e afetiva (Leal, 2000) é uma visão que não 
inclui a diversidade de adolescências que podem ser vividas nem as possibilidades que os diversos contextos fornecem para o seu desenvolvimento. A possibilidade da adolescência poder ser vivida como fase de transição para a vida adulta é exclusiva da classe média, já que os adolescentes de classes populares não terão esta oportunidade. Pelo contrário, para eles, a adolescência pode ser uma fase em que terão que assumir a responsabilidade da sua vida, nomeadamente, na necessidade de arranjar trabalho e definir o seu relacionamento afectivo. Esta posição é partilhada por Heilborn et al. (2002). Segundo as pesquisadoras analisar o impacto da gravidez nas trajetórias escolar e profissional dos adolescentes, requer um exercício de relativização destas instâncias nas vidas destes jovens das diferentes classes sociais. $\mathrm{O}$ fenômeno do prolongamento da juventude não parece ter grande impacto para o grupo de baixo nível socioeconômico, no qual há uma preocupação dominante com a sobrevivência, o que influencia a sua relação com a escola. Nestes grupos, a escola é encarada como uma necessidade provisória até permitir o acesso a um trabalho que assegure a subsistência e permita contribuir para o orçamento familiar. Por esse motivo, a análise dos casos de gravidez adolescente deve contemplar o histórico e o retrato da situação referente à relação destes jovens com a escola, o trabalho e a família e as múltiplas variáveis associadas a um percurso individual de vida.

Dados da pesquisa GRAVAD (2006) reforçam esta perspectiva. Os valores apresentados revelam que $42,1 \%$ das jovens com menos de 20 anos que tiveram filhos, já não frequentavam a escola à data da gravidez. E 62,6\% das adolescentes, no nascimento do primeiro filho, encontravam-se já fora do mercado de trabalho e assim se mantiveram. Nesses casos os fatores de risco já estavam presentes, uma vez que o abandono escolar e a ausência de profissionalização, impossibilitam o acesso ao mercado de trabalho, prejudicando o auto-sustento (WHO, 2004). Salientase que, em relação ao abandono escolar, é precipitado afirmar que a gravidez estaria na sua origem já que, segundo um estudo de Figueró (2002), parte das gestantes e mães adolescentes já haviam abandonado a escola previamente à gravidez. Pelo contrário, jovens com bons níveis de desempenho escolar e aspirações acadêmicas têm maior probabilidade de adiar a sua iniciação sexual e buscar meios contraceptivos, assim como, em recorrer ao aborto, no caso de engravidarem (Levandowski \& Piccinini, 2004; Manlove, 1998).

Quanto a características da gravidez na adolescência, Gama, Szwarcwal e Leal (2002) mostraram, em um estudo comparativo entre três grupos de gestantes, sendo um deles de adolescentes, que há diferenças na forma como estas percebem e conduzem a sua gestação. Especificamente, indicaram que o desejo daquela gravidez e o número de consultas pré-natais foi menor no grupo de gestantes adolescentes comparativamente às restantes. Por sua vez, a incidência de partos prematuros e de bebês com baixo peso ao nascer neste grupo foi superior em relação aos outros.

Para além disto, Cabral (2003) revelou que um outro fator de risco da parentalidade na adolescência é a imaturidade psíquica dos jovens pais, os quais se revelam pouco contingentes às necessidades desenvolvimentais do bebê, bem como, para educar e criar uma criança. Tal imaturidade pode deixar a criança mais propensa a contrair doenças infectocontagiosas ou, até mesmo, a sofrer acidentes. A interação que a mãe adolescente estabelece com o seu bebê tem características específicas: dificuldade em apreender o bebê como uma entidade distinta de si, com comportamentos e necessidades próprias, o que leva ao estabelecimento de uma relação mais dirigida ao plano da fantasia que da realidade. Em comparação às mães adultas, assiste-se, por parte das mães adolescentes, a um menor número de ações contingentes às necessidades do bebê, oferecendo-lhes menos atividades de estimulação, pouca comunicação e um maior número de comportamentos de indiferença relativamente aos seus pedidos. Não obstante, verificase que as mães adolescentes oferecem mais contato físico aos seus bebês que as mães adultas (Figueiredo, 2001). No contexto da proteção, o estudo de Figueiró (2002) identificou uma menor prevalência de gravidez e de maternidade entre as adolescentes cujo pai e mãe residiam no domicílio e entre as que estavam trabalhando. Verificou também que o envolvimento familiar em grupos religiosos é uma variável que favorece o adiamento do início da vida sexual/reprodutiva na adolescência.

Uma gravidez não planejada revela invariavelmente a exposição a, pelo menos, uma situação de risco, o sexo sem preservativo/proteção. Essa atitude, poderá refletir, por parte dos adolescentes, a gravidez como algo gratificante, do ponto de vista pessoal e afetivo. É um momento no qual as adolescentes imaginam e projetam o papel de mãe, frequentemente, com pouca maturidade, de forma positiva, irrealista e idealizada, identificando a tarefa de cuidar de um bebê como fácil e divertida (Jaccard, Dodge \& Dittus, 2003; Figueiredo, 2001). Para além da gravidez propriamente dita, a discussão em questão coloca em foco uma alteração no ciclo de desenvolvimento destes adolescentes pais e mães, a 
partir do nascimento da criança. Nesse sentido, buscase uma reflexão a longo prazo do fenômeno da gravidez na adolescência.

O surgimento desta gravidez compromete também a capacidade de autonomização, por parte da adolescente, relativamente às figuras parentais (tarefa que caracteriza o período da adolescência) introduzindo uma certa ambivalência na relação, já que ao ser mãe há uma precipitação desta autonomia, embora na maioria dos casos, persista a dependência relativamente aos seus pais, nomeadamente a nível econômico (Figueiredo, 2001). Contudo, também se verifica que quando a jovem possui capacidade maturativa, a gravidez permite a aquisição de ganhos significativos, nomeadamente relativamente à construção da identidade sexual e de uma autonomização relativamente aos seus próprios pais (Figueiredo, 2001). Neste sentido, a gravidez poderá ser considerada como um fator protetor do ponto de vista desenvolvimental da adolescente.

Ao avaliar adolescentes em situação de risco, Pantoja (2003) aponta a maternidade como uma forma de ascensão social e uma passagem para a vida adulta, especialmente, para garantir a estima de outras pessoas e um futuro melhor para e através do filho. Gontijo e Medeiros (2004) discutiram uma série de estudos que tratam das percepções da gravidez para adolescentes em situação de risco pessoal e social e identificaram que a maternidade pode ser vista de forma positiva para essas adolescentes, pois a maternidade adquire um papel central, oferecendo novas possibilidades de reconhecimento e atuação social.

Discutir a gravidez na adolescência daqueles que vivenciam situações de pobreza ultrapassa a simples identificação dos riscos, exatamente pelo fato de que já existem riscos envolvidos como a baixa condição socioeconômica. É necessário prescindir de uma teoria que possa avaliar a complexidade da gravidez desse adolescente que vivencia o risco constantemente.

Este artigo apresenta a proposta de análise desse fenômeno a partir da Abordagem Bioecológica do Desenvolvimento Humano (Bronfenbrenner \& Evans, 2000; Bronfenbrenner \& Morris, 1998). Bronfenbrenner formulou uma teoria que contempla o desenvolvimento humano, a partir dos processos de interação que estabelece com outras pessoas, objetos e símbolos em diferentes contextos ecológicos através do tempo. Esse modelo teórico-metodológico compreende o desenvolvimento humano através das mudanças e da estabilidade produzida nas características biopsicológicas da pessoa ao longo de sua vida. Essa concepção possibilita analisar a pessoa como um ser ativo e dinâmico, que interage com o contexto que vivencia, modificando e sendo modificada por ele.

A partir dessa ótica, a gravidez na fase da adolescência vivenciada pelos adolescentes e jovens precisa ser compreendida através da interação de quatro núcleos inter-relacionados: o processo, a pessoa, o contexto e o tempo. O processo é responsável pelo desenvolvimento e envolve os processos proximais, caracterizados pela interação recíproca progressivamente mais complexa de um ser humano ativo, biopsicologicamente em evolução, com as pessoas, objetos e símbolos presentes no seu ambiente imediato (Bronfenbrenner \& Morris, 1998). A pessoa é analisada através de suas características determinadas biopsicologicamente e aquelas construídas na sua interação com o ambiente (Bronfenbrenner \& Morris, 1998).

Para avaliar o ambiente ecológico, Bronfenbrenner e Morris (1998) sugerem a análise de quatro níveis ambientais, denominados como microssistema, mesossistema, exossistema e macrossistema. O microssistema é caracterizado como um contexto no qual são estabelecidas relações e atividades face-a-face e onde operam os processos proximais que produzem, sustentam o desenvolvimento e no qual se assume um papel social. Neste caso, seria constituído pela grávida adolescente e a sua rede direta de apoio, como pais, namorado, amigos, escola. Ao longo do ciclo de vida da pessoa, as suas relações se tornam mais complexas e outros microssistemas podem fazer parte do ambiente ecológico como a escola e a rede de apoio social e afetivo. Esse conjunto de microssistemas forma o mesossistema. O exossistema envolve os ambientes que a pessoa não frequenta como um participante ativo, mas que desempenham uma influência indireta sobre o seu desenvolvimento (Bronfenbrenner, 1979/1996). No contexto da gravidez adolescente poderiam ser identificadas, por exemplo, as estruturas de ensino e saúde. O macrossistema é composto pelo padrão global de ideologias, crenças, valores, religiões, formas de governo, culturas e subculturas presentes no cotidiano das pessoas que influenciam seu desenvolvimento (Bronfenbrenner, 1979/1996). Como a percepção social e cultural sobre este fenômeno que influenciarão, embora indiretamente, a forma como a jovem, a sua família e a sociedade lidam com este acontecimento.

Por fim, o modelo bioecológico privilegia o tempo, examina a influência para o desenvolvimento humano de mudanças e continuidades que ocorrem ao longo do ciclo de vida (Bronfenbrenner, 1986). Nesse contexto pode ser identificado o desenvolvimento 
destas jovens mães e dos seus filhos, e também a qualidade interacional que estabelecem com aqueles com quem se relacionam. A análise do tempo focaliza a pessoa em relação aos acontecimentos presentes em sua vida, os mais próximos até os mais distantes, como grandes acontecimentos históricos, por exemplo. Bronfenbrenner e Morris (1998) ressaltam que as mudanças que ocorrem através do tempo, nas quatro propriedades do modelo bioecológico, não são apenas produtos, mas também produtores da mudança histórica.

Tendo em vista esse modelo teóricometodológico, este artigo tem como objetivo analisar o fenômeno da gravidez para adolescentes que vivem em situação de pobreza e identificar os fatores de risco e os fatores de proteção associados a este acontecimento. Tal escolha se justifica devido à necessidade de compreender a pessoa em foco, no caso a adolescente, a sua própria interpretação da realidade e as interações estabelecidas com seu ambiente ecológico.

\section{MÉTODO}

\section{Delineamento e Participantes}

Este estudo teve um delineamento transversal, de caráter analítico, no qual participaram 1015 jovens estudantes de ambos os sexos em situação de pobreza da cidade de Porto Alegre. As idades variaram de 14 a 24 anos (média de 15,98; $d p=1.98$ ), sendo $48 \%$ dos participantes do sexo masculino e $52 \%$ do sexo feminino.

Os participantes deste estudo compõe o banco de dados da Pesquisa Nacional de Fatores de Risco e Proteção da Juventude Brasileira. Os participantes desta pesquisa pertenciam ao nível socioeconômico baixo e frequentavam escolas e da rede pública e institutos de apoio de sete capitais (Porto Alegre, Recife, São Paulo, Belo Horizonte, Brasília, Campo Grande e Manaus) e três cidades brasileiras (Arcos, Minas Gerais; Presidente Prudente, São Paulo; e Maués, Amazonas).

\section{Procedimentos}

\section{Procedimento de amostragem}

A partir de dados do IBGE (Censo 2000), foram levantados indicadores das condições sóciodemográficas de cada bairro da cidade de Porto Alegre. Cinco indicadores básicos foram escolhidos para analisar a situação de cada um dos bairros: rendimento e nível de alfabetização do chefe da família, situação do domicílio (tipo de construção), existência de água encanada e rede de esgoto.

Foram listados os bairros que se encontravam abaixo de uma linha de corte, para, no mínimo, dois indicadores. A partir do número de bairros que foram selecionados, foi realizado um cálculo para obter a proporção de bairros por regiões geográficas da cidade (centro, norte, sul e leste) ${ }^{1}$. A partir deste número, foi feito um sorteio de dez bairros segundo a proporção para cada zona da cidade. A partir de duas listas de escolas (Municipais e Estaduais) foi realizado um novo sorteio para selecionar uma escola para cada bairro. Todos os alunos dentro da idade selecionada foram convidados a participar do estudo, obtendo-se uma média de 100 estudantes por escola.

\section{Instrumentos e Medidas}

Foi utilizado um questionário para levantamento de fatores de risco e proteção, produzido para o estudo "Juventude Brasileira" (Koller, Cerqueira-Santos, Morais, \& Ribeiro, 2005). O instrumento consta de 109 questões de múltipla escolha e investiga aspectos sobre a caracterização biosociodemográfica dos participantes, assim como sobre as temáticas de educação, saúde (incluindo drogas e sexualidade), trabalho, violência, lazer, religiosidade, rede de apoio social, humor, auto-estima e auto-eficácia. O instrumento foi respondido individualmente, em sala de aula e o tempo de preenchimento foi, em média, de uma hora e meia. Para este estudo foram utilizados os dados sobre comportamento sexual, como, uso de preservativo, gravidez e contracepção.

\section{Análise de Dados}

Os dados foram tabulados no SPSS for Windows e passaram por análises descritivas, freqüência e percentual; e análises bivariadas, testes $T$ de Student e Qui-Quadrado.

\section{Aspectos Éticos}

Os aspectos éticos que garantem a integridade dos participantes deste estudo foram assegurados. Além do termo de consentimento individual, foi dada a garantia de sigilo das informações pessoais, assim como foi disponibilizada a assistência do grupo de pesquisa, caso algum participante necessitasse de apoio psicológico provocado pela lembrança negativa de algum dos aspectos investigados (Resolução 016/2000

\footnotetext{
A região oeste de Porto Alegre é ocupada pelo Rio Guaíba, uma vez que esta divisão é feita tendo o centro comercial da cidade como referência.
} 
CFP). O projeto foi aprovado pelo Comitê de Ética em Pesquisa da UFRGS. O presente estudo foi aprovado pelo Comitê de Ética do Instituto de Psicologia da UFRGS, com o protocolo n ${ }^{\circ}$ 205/466.

\section{RESULTADOS}

Os principais resultados mostram que $42,6 \%$ dos jovens responderam já ter tido a primeira relação sexual. Desses, 63,9\% tinham na época idades entre os 14 e 17 anos. Do total da amostra, 47,7\% relataram manter uma vida sexual ativa, sendo que mais da metade destes $(55,4 \%)$ afirma ter tido sua primeira experiência antes dos 15 anos de idade. Entre o grupo de participantes masculinos, 64,2\% já tiveram alguma relação sexual. Já no grupo feminino esse índice cai para 43,3\% (ver Tabela 1). Houve diferença significativa na idade média da primeira relação sexual por sexo, sendo 13,64 anos $(d p=1,7)$ para os homens e 14,79 anos $(d p=1,4)$ para mulheres $(t=7,97$; $g l=492 ; p<.001)$.

Tabela 1. Primeira Relação Sexual para Ambos os Sexos

\begin{tabular}{|c|c|c|c|c|c|c|}
\hline \multirow{3}{*}{$\begin{array}{c}\text { Primeira } \\
\text { Relação } \\
\text { Sexual }\end{array}$} & \multicolumn{4}{|c|}{ Sexo } & \multirow{3}{*}{$\begin{array}{c}\text { Estatística } \\
\text { teste } \\
\chi^{2}\end{array}$} & \multirow{3}{*}{$p$ valor } \\
\hline & \multicolumn{2}{|c|}{ Masculino } & \multicolumn{2}{|c|}{ Feminino } & & \\
\hline & $\mathbf{n}$ & $\%$ & $\mathbf{n}$ & $\%$ & & \\
\hline Sim & 314 & 64,6 & 229 & 43,3 & \multirow{2}{*}{14,98} & \multirow{2}{*}{$<0,001 *$} \\
\hline Não & 173 & 35,4 & 298 & 56,7 & & \\
\hline
\end{tabular}

* significativo $\mathrm{p}<0,05$

Analisando as diferenças de sexo para o uso da camisinha, encontrou-se que há diferença significativa entre homens e mulheres para os dois tipos de uso (Tabela 2). Homens afirmaram maior frequência de uso da camisinha em ambos os casos (evitar HIV/AIDS e como método contraceptivo). A diferença de sexo foi mais acentuada para o uso como método contraceptivo, do que como método para evitar HIV/AIDS. Considerando que as mulheres podem utilizar outros métodos contraceptivos, detectou-se a diferença significativamente maior para elas utilizando outros métodos. No entanto, este resultado aponta para o fato de que as mulheres podem estar mais vulneráveis à contaminação pelo HIV/AIDS.

Quanto ao uso de métodos anticoncepcionais entre os jovens sexualmente ativos (ver Tabela 2), $8,8 \%$ disseram nunca usar, $15,6 \%$ usa às vezes e $75,6 \%$ disse usar sempre. Como esperado, houve diferença entre homens e mulheres para o não uso de anti-concepcionais $(12,6 \%$ para eles e $4,1 \%$ para elas, $\left.\chi^{2}=14,79 ; p=0,001\right)$.

Tabela 2. Uso de Método Anti-Concepcional por Sexo do Participante

\begin{tabular}{|c|c|c|c|}
\hline \multirow{3}{*}{ Método } & \multicolumn{3}{|c|}{ Sexo } \\
\hline & & Masculino & Feminino \\
\hline & & n $\%$ & n $\%$ \\
\hline \multirow{3}{*}{ Anti-concepcional } & Nunca & 4012,6 & 94,1 \\
\hline & Às vezes & 3711,9 & 4519,5 \\
\hline & Sempre & 9675,5 & 17576,4 \\
\hline
\end{tabular}

$\chi^{2}=14,79 ; p=0,001$

Mais de $11 \%$ (55) dos participantes já estiveram grávidas ou já engravidaram a sua parceira, sendo que $31,7 \%$ destes não possuem nenhum filho vivo. Dos abortos registrados, $21,8 \%$ (12) disseram ter sofrido aborto natural e $10,9 \%$ (6) sofreram aborto provocado (ver Tabela 3). Não houve registro de casos de morte no parto.

Tabela 3. Episódios de Gravidez e Aborto

\begin{tabular}{lcccc}
\hline \multicolumn{1}{c}{ Episódios } & $\begin{array}{c}\text { Nenhum(a) } \\
(\mathbf{\%})\end{array}$ & $\begin{array}{c}\text { Uma } \\
(\mathbf{\%})\end{array}$ & $\begin{array}{c}\text { Duas } \\
(\mathbf{\%})\end{array}$ & $\begin{array}{c}\text { Três ou mais } \\
(\mathbf{\%})\end{array}$ \\
\hline $\begin{array}{l}\text { História de } \\
\text { gravidez* }\end{array}$ & 84,9 & 10,1 & 1,8 & 1,3 \\
Filhos vivos & 31,7 & 54,4 & 11,7 & 2,3 \\
Abortos naturais & - & 83,9 & 8,9 & 5,4 \\
$\begin{array}{l}\text { Abortos } \\
\text { provocados }\end{array}$ & - & 52,9 & 17,6 & 11,8 \\
\hline
\end{tabular}

*Alguns casos apresentaram missing

Dentre os participantes que já passaram por pelo menos um episódio de gravidez, mais de 68\% relataram o nascimento dos filhos. Embora a maioria das mães e pais tenha afirmado morar com seu próprio filho $(68,2 \%)$, os dados revelaram que esses jovens ainda mantêm relações de dependência econômica e domiciliar com as famílias de origem para o cuidado do filho. O pai ou a mãe, os avós e a família adotiva foram indicados como principais responsáveis pela moradia dos filhos (ver Tabela 4).

Tabela 4. Com Quem os Filhos Moram

\begin{tabular}{lcc}
\hline Convivem & $\mathbf{n}$ & $\mathbf{\%}$ \\
\hline Comigo & 26 & 66,5 \\
Com o pai/mãe & 8 & 20,5 \\
Avós & 2 & 5,2 \\
Família Adotiva & 1 & 2,5
\end{tabular}


Não Sei

$2 \quad 5,2$

A Tabela 5 expõe os percentuais encontrados para as consequências geradas após a gravidez. Sentimentos positivos foram expressos pela maior parte dos jovens através da identificação desse momento como importante $(74,5 \%)$ e produtor de orgulho (56\%). Ao mesmo tempo, salientaram que a gravidez na adolescência acarreta preocupação, vergonha, desemprego, necessidade de trabalho, interrupção nos estudos e casamento forçado.

Tabela 5. Consequências da Gravidez

\begin{tabular}{lccc}
\hline \multicolumn{1}{c}{ Consequências } & Discordo & $\begin{array}{c}\text { Nem concordo } \\
\text { nem discordo }\end{array}$ & Concordo \\
\hline Gravidez Importante & $14,5 \%$ & $10,9 \%$ & $74,5 \%$ \\
Gravidez Desejada & $43,4 \%$ & $17 \%$ & $39,6 \%$ \\
Trouxe Vergonha & $62,5 \%$ & $16,7 \%$ & $20,8 \%$ \\
Trouxe Preocupação & $32,7 \%$ & $11,5 \%$ & $55,8 \%$ \\
Gravidez Escondida & $60,9 \%$ & $6,5 \%$ & $32,6 \%$ \\
Trouxe Orgulho & $20 \%$ & $24 \%$ & $56 \%$ \\
Trouxe Desemprego & $84,1 \%$ & $4,5 \%$ & $11,4 \%$ \\
Trouxe Casamento & $69 \%$ & $7,1 \%$ & $23,8 \%$ \\
Trouxe Casamento Forçado & $88,4 \%$ & $7 \%$ & $4,7 \%$ \\
Necessidade de Trabalho & $63 \%$ & $6,5 \%$ & $30,4 \%$ \\
Parar de estudar & $67,3 \%$ & $4,1 \%$ & $28,6 \%$ \\
\hline
\end{tabular}

\section{DISCUSSÃO}

Os resultados obtidos nesta pesquisa revelam que não é possível descrever a gravidez durante a adolescência de forma determinística e causal, já que ela é o produto de conjunção de múltiplas variáveis (Jacard et al., 2003). Segundo os dados obtidos e a diversidade de resultados recolhidos confirma-se o posicionamento de certos autores que descrevem a gravidez adolescente como um fenômeno que envolve diferentes fatores de risco (Canavarro \& Pereira, 2001; Jacard, Dodge, \& Dittus, 2003; Pantoja, 2003). A influência destas variáveis depende da perspetiva utilizada na análise do fenômeno. Este fato corrobora o posicionamento da Abordagem Bioecológica do Desenvolvimento Humano (Bronnfenbrener \& Morris, 1996), no qual se descreve a importância do contexto no desenvolvimento humano, na forma como o sujeito interage com aqueles que o rodeiam e com o meio em que está inserido. Logo, o impacto de cada acontecimento depende da composição dos microssistemas em que se está inserido e a interação destes com o mesossistema, exossistema e macrosssitema. Neste sentido consideramos que a pertença a um nível socioeconômico baixo poderá traduzir um micro e mesossistema mais carenciado ao nível das informações sobre sexualidade, cuidados de saúde e importância de contracepção, assim como, no acesso aos serviços de saúde e, por esse motivo, um contexto de maior risco.

Esse fato poderá ajudar a entender os dados exploratórios que revelam a idade da primeira relação sexual como abaixo dos índices apontados pelo Ministério da Saúde (2005). Este dado está de acordo com a previsão da redução da idade para a primeira relação sexual e confirma a tendência apontada por outros estudos (Szwarcwald, Júnior, Pascom \& Júnior, 2004) e a baixa idade para primeira relação sexual parece estar associada ao nível socioeconômico dos jovens, corroborando estatísticas do WHO (2005) e do Ministério da Saúde (2002), assim como a hipótese defendida por Waystaff, Delameth e Havens (1999) de que a população empobrecida está mais vulnerável aos comportamentos sexuais de risco.

Inúmeras justificativas são oferecidas para explicar essas relações. Reiteradamente a literatura salienta a pobreza, a baixa escolaridade e a baixa idade como fatores de risco para a ocorrência da gravidez em adolescentes que vivem em situação econômica desfavorável (Borges, \& Schor, 2005; Brandão, \& Heilborn, 2006; Gama, Szwarcwal, \& Leal, 2002). Certamente, a pobreza potencializa a exposição aos riscos que, de alguma forma ameaçam o desenvolvimento saudável, no entanto, focalizar a explicação da gravidez na adolescência exclusivamente nessa questão impede um olhar ecológico para esse fenômeno. Os dados encontrados revelam que o ambiente ecológico do desenvolvimento humano envolve diferentes sistemas inter-relacionados que se afetam mutuamente. O baixo nível socioeconômico, por exemplo, compõe apenas um fator do modelo bioecológico, composto pela pessoa, processo, tempo e contexto.

Ao analisar a pessoa verifica-se que as características do indivíduo também contribuem para a manifestação da sexualidade dos jovens. Os resultados obtidos apontam a diferença de sexo, confirmando a manutenção da divergência cultural na forma como moças e rapazes lidam com a sua vida sexual (Antunes, Peres, Paiva, Stall, \& Hearst, 2002). A entrada da menina na adolescência é marcada pela menarca, um evento biológico e temporalmente bem localizado que abre precedentes para discussão e interpretação cultural. Já para os meninos, o marcador 
não é tão preciso, sendo as experiências sexuais um grande definidor do desenvolvimento adolescente, reforçado pelo grupo e, muitas vezes, pela família. Essas expectativas e os valores sociais podem influenciar a forma como a própria pessoa lida com o seu próprio desenvolvimento. Talvez essas atitudes pessoais favoreçam a iniciação sexual mais precoce dos meninos, os quais revelaram iniciar as relações sexuais um ano mais cedo do que as meninas. No entanto, essa diferença não diminui a exposição aos riscos envolvidos no comportamento sexual delas. Pelo contrário, os rapazes afirmam utilizar com maior frequência métodos anti-concepcionais, especialmente a camisinha, quando comparados às moças. Diante dessas informações constata-se que as participantes do sexo feminino estão mais propensas à gravidez e as doenças sexualmente transmissíveis. Reis e OliveiraMonteiro (2007) encontraram resultados semelhantes sobre a iniciação sexual, apontando que os jovens do sexo masculino mostram-se mais precoces e mais favoráveis ao uso de preservativos.

Esses adolescentes precisam ser vistos como pessoas em sua totalidade, com suas histórias, suas experiências e suas vivências sexuais. A forma como os adolescentes interpretam as suas interações sexuais revelam os processos proximais que estabelecem com os seus parceiros. Na maioria das vezes, as relações sexuais são fruto de interações que não são significativas e tampouco duradouras, trazendo muitas vezes resultados disfuncionais. Neste estudo, esse tipo de efeito pode ser representado pela ocorrência do aborto natural e provocado, soluções imediatas da gravidez indesejada identificadas por $10,9 \%$ dos entrevistados. Pesquisadores sugerem que a proporção do aborto é maior entre os adolescentes com idade inferior a 20 anos (Aquino et al., 2003; Vieira, Goldberg, Saes \& Dória, 2007). Por outro lado, a transição ecológica imposta pela gravidez, força a menina a assumir um outro papel nas relações interpessoais e promove o seu engajamento na nova atividade: ser mãe e manter o cuidado de seu filho.

Tal como se pode ver nos resultados, muitos adolescentes decidem manter a gestação até o final. $\mathrm{O}$ nascimento de um filho incrementa o processo de transição para a vida adulta, através da emergência de novos papéis como mãe e pai. Pantoja (2003) salienta que a maternidade garante um passaporte para a vida adulta e garante o cuidado através de outras pessoas. Nesse sentido, a gravidez acaba por funcionar como um fator protetor para essa adolescente. A proteção pode ser justificada tanto pelo plano de vida que passa a adquirir quanto pela ascenção e proteção social que passa a ter no interior da família e que lhe permite atuar nas suas circunstâncias, diminuindo as adversidades e dificuldades de que é alvo. Este dado pode ser ilustrado pelo fato da amostra ser o principal cuidador do seu filho e uma larga maioria descreve a gravidez como um acontecimento importante da sua vida. Alternativas a esse cuidado foram retratadas através do sustento oferecido pelos avós, pela figura do pai ou da mãe ou ainda por família adotiva. Essa informação revela que esses adolescentes ainda mantêm relações de dependência econômica com as famílias de origem e famílias substitutas para o cuidado do filho (Leal, 2000; Figueiredo, 2001).

Todas essas mudanças ocorrem em um meio ambiente ecológico Bronfenbrenner (1979/1996), composto por uma série de contextos, desde os mais próximos até os mais distantes. Talvez a mudança mais significativa ocorra no microssistema, uma vez que os padrões de atividades e papéis experienciados nos ambientes mais próximos se modificam. $\mathrm{O}$ suporte emocional que o microssistema familiar pode oferecer é de extrema importância para reforçar a responsabilidade que o adolescente deve assumir pelo filho. No entanto, pesquisas têm apontado que as avós e os avôs vivenciam conflitos na delimitação desses papéis e acabam por assumir a responsabilidade pelo cuidado infantil (Falcão \& Salomão, 2005; Silva \& Salomão, 2003). Os pesquisadores citam também outras situações típicas vivenciadas pelos avós diante da maternidade que podem envolver a vergonha e a falta de confiança na maturidade do adolescente até oferecer apoio e confiança para a responsabilidade do cuidado.

Os adolescentes provenientes de microssistemas familiares e educativos descomprometidos com o cuidado e a proteção terão dificuldade para efetivar relações significativas e protetivas. Nesses casos, a gravidez pode surgir como facilitador para a saída do microssistema familiar fragilizado e para a construção de um novo lar. Reis e Oliveira-Monteiro (2007) ao avaliarem as motivações para uma futura gravidez, encontraram que as carências afetivas e materiais são indicadores do desejo de ter um filho. Os autores ressaltaram que as meninas mencionam com maior frequência a vontade de ter a própria família, as brigas familiares e desejo de não se sentir só como principais motivadores de uma gestação, enquanto os meninos citaram a falta de oportunidades e de lazer.

Embora a construção de um novo lar possa assumir um fator protetivo a princípio, é preciso atentar para os novos riscos que poderão surgir a partir dessa transição ecológica, especialmente, porque essa passagem é mais efetiva e saudável na medida que a adolescente grávida se sente apoiada e tem a 
participação de suas relações significativas neste processo (Bronfenbrenner, 1979/1996). Quando isso não acontece, novos fatores de risco podem emergir, desde as dificuldades impostas pela gravidez até as implicações sociais, econômicas e afetivas da maternidade durante a adolescência. Ao avaliar a concepção das próprias adolescentes que vivem em situação de pobreza verificou-se que a gravidez trouxe modificações também no mesossistema. Para Bronfenbrenner (1979/1996), o mesossistema referese aos elos e aos processos entre dois ou mais ambientes, nos quais os indivíduos se desenvolvem, isto é, a interação entre os diversos microssistemas. Os resultados revelaram que a necessidade de emprego e abandono do estudo modificaram e ampliaram os microssistemas nos quais estavam inseridas.

Nesse sentido, a escola pode assumir um importante fator de proteção para a gravidez na adolescência. A interação entre pares, a presença de professores comprometidos com a formação plena somado ao apoio e a presença familiar podem compor o mesossistema dos adolescentes. Uma importante discussão avalia se o abandono dos estudos é provocado pela gestação ou se o prévio abandono é fator de risco para a gravidez na adolescência. Figueró (2002) observou que parte das gestantes e mães adolescentes abandonou a escola previamente à gravidez. Já os dados da WHO (2004) apontam um alto índice de jovens grávidas que abandonam a escolarização dificultando a futura inserção no mercado de trabalho (WHO, 2004). Estes dados confirmam os estudos que demonstram como o surgimento da gravidez compromete a capacidade de autonomização, por parte da adolescente, relativamente às figuras parentais, persistindo a dependência relativamente aos seus pais, nomeadamente a nível econômico (Figueiredo, 2001).

No Brasil, as meninas estudam mais do que os meninos, então o impacto da gravidez deve ser maior entre elas. Esse impacto pode ser sentido mesmo antes do nascimento do bebê, pois a modificação do corpo da menina torna visível o seu comportamento sexual de risco e pode gerar dificuldades no contexto escolar. Por outro lado, certos autores, referem a ambição acadêmica e o rendimento escolar como variáveis que influenciam o adiamento da iniciação sexual, assim como o maior uso de contracepção, mas também maior recurso ao aborto, em caso de gravidez não planejada (Levandowski \& Piccinini, 2004; Manlove, 1998).

Segundo Santos Júnior (1999) os professores são mal preparados, para conduzir a discussão sobre o comportamento sexual e acabam por ter condutas discriminatórias, geralmente tentando excluir das salas de aula as meninas gestantes, com o intuito de não servirem de "mau exemplo" às outras colegas. Essa discriminação pode favorecer o desligamento das meninas da sala de aula mostrando o quanto a escola pode ser um fator de risco para essa população. É emergente que esse mesossistema realize uma avaliação do seu papel frente a essas situações. A escola deveria compor a rede protetiva dos adolescentes, promovendo ações preventivas relacionadas à sexualidade. Excluir ou expulsar os jovens que vivenciam essa situação não resolve essa questão, assim como não diminui o número de gravidez nessa fase do desenvolvimento. Ao contrário, o vínculo com a escola fortalece a formação e aumenta a capacidade de competição no mercado de trabalho futuro. Entretanto, essa realidade não é visível no sistema educacional e acaba por fortalecer o vínculo dos adolescentes com outros contextos como, por exemplo, o trabalho.

A necessidade de trabalho surge para suprir as despesas que um filho demanda. Os resultados indicaram que a maioria dos participantes discordou sobre a necessidade de trabalho após a gravidez. Cabe relembrar que essa amostra é composta por adolescentes que vivem em situação de pobreza. Heilborn et al. (2002) apontam que os trabalhos não acontecem apenas nos casos vinculados à gravidez, ao contrário, o fato de pertencer a um grupo social economicamente desfavorável torna-se um fator de risco mais precoce para inserção na atividade laboral.

Para Bronfenbrenner, nenhum aspecto pessoal ou social pode exercer influência sobre o desenvolvimento de forma isolada, por esse motivo, o exossistema, caracterizado como um ou mais ambientes nos quais a pessoa não participa face-aface, mas cujas decisões tomadas, direta ou indiretamente, também influenciam na vida sexual dos adolescentes (Bronfenbrenner, 1979/1996). As comunidades mais empobrecidas dependem da ação direta de órgãos governamentais e nãogovernamentais como, por exemplo, ações comunitárias e ações planejadas pela Secretária da Saúde. A escassez de uma rede de apoio social e comunitária efetiva é representada, muitas vezes, pela inexistência de um posto de saúde preparado para intervenção e ações preventivas na saúde sexual e reprodutiva. Tal fato contribui ainda mais com a vulnerabilidade sexual da população inserida nessas comunidades.

A importância do cuidado a saúde desses reforça a necessidade de um exossistema ativo, capaz de propor políticas públicas preventivas e eficazes sobre 
a sexualidade e a reprodução humana. A falta de recursos financeiros, acrescida da escassez de redes de atendimento no mesossistema, expõe os adolescentes a comportamentos sexuais de risco e a interrupção da gravidez. Diante desse cenário, é visível como as decisões tomadas no exossistema e a inexistência de um mesossistema capaz de oferecer uma rede de apoio social e comunitária podem interferir no desenvolvimento sexual seguro e protetivo dos adolescentes.

Outro sistema ecológico importante na discussão dessa problemática são os aspectos macrossistêmicos, ou seja, as ideologias, as crenças, os valores, as culturas e subculturas presentes no cotidiano das pessoas que influenciam no desenvolvimento (Bronfenbrenner, 1979/1996). A gravidez na adolescência tem sido tratada como um problema de saúde pública (Gontijo \& Medeiros, 2004). Essa percepção não tem sido corroborada quando o alvo dos estudos são as próprias adolescentes grávidas. Uma séire de estudos desenvolvidos com adolescentes em situação de risco tem identificado diferentes significados revelando valores, culturas e crenças dessa população (Brandão \& Heilborn, 2006; Cabral, 2003; Gontijo \& Medeiros, 2004, Reis \& OliveiraMonteiro, 2007).

O presente estudo solicitou aos próprios adolescentes a avaliação das consequências geradas com o evento da gravidez e uma diversidade de argumentos foi colocada em pauta. Observando-se que a existência da gravidez adolescente não é um fenômeno determinístico e que a forma como é vivida depende das características pessoais (Canavarro \& Pereira, 2001; Jacard, Dodge, \& Dittus, 2003) diferentes significados foram atribuídos. Os resultados revelaram não só aspectos macrossistêmicos como aspectos do tempo, quarto componente do modelo bioecológico. Os sentimentos positivos foram expressos pela maior parte dos jovens através da identificação desse momento como importante. Embora a gravidez não tenha sido planejada, foi desejada por um número significativo e produtora de orgulho. Apesar disso, um número relevante de adolescentes mencionaram o não desejo da sua gravidez, o facto de a esconderem de outras pessoas, assim como, terem vergonha desse acontecimento. Por outro lado, muitas das jovens manifestam o desejo daquela gravidez, descrita como um acontecimento importante da sua vida pela maioria delas. O que vai ao encontro do referido na literatura, na qual a gravidez adolescente é descrita como emergente na ausência de outros projetos (Pantoja, 2003; Pesquisa
GRAVAD, 2006) e de relações sociais consideradas pouco satisfatórias.

Contudo, o fato da maioria da população amostral jovens ter referido o acontecimento como gerador de preocupação, demonstra uma consciência da responsabilidade que esse novo papel desempenhará na sua vida. Segundo os dados apurados, registou-se que a maioria das mães adolescentes vive com os seus próprios filhos, evidenciando que estas assumem a sua maternidade e a educação do seu filho para si. As principais mudanças decorrentes da gravidez envolvem a necessidade de trabalhar, a interrupção dos estudos e o casamento. Nos casos em que a jovem possui capacidade maturativa, a gravidez poderá permitir a aquisição de ganhos significativos, nomeadamente relativamente à construção da identidade sexual e de uma autonomização relativamente aos seus próprios pais (Figueiredo, 2001).

\section{CONCLUSÕES}

Esse artigo teve como objetivo explorar alguns dos fatores de risco e proteção associados a uma população adolescente porto-alegrense com experiência de gravidez. Com este estudo procurou refletir-se sobre a forma como esta situação de gravidez pode ser vivida, demonstrando que a sua existência adquire um curso individual e único, para cada adolescente, dependendo das características contextuais em que está inserida. Por esse motivo, a gravidez durante a adolescência, por si só, não poderá ser descrita como fator de risco ou de proteção. Além disso, pretendeu atentar-se para a complexidade que envolve a sexualidade na adolescência quando somada a situação de pobreza. A situação econômica desfavorável que muitos jovens enfrentam aumenta a vulnerabilidade e a exposição aos comportamentos sexuais de risco. Isso não significa que a condição socioeconômica, o não uso de métodos anticoncepcionais seja a causa para a iniciação sexual precoce e a gravidez na adolescência e vice-versa. É preciso ampliar o olhar para esse fenômeno, ao invés de apontar, variáveis isoladas que sozinhas não suportam o conjunto de fatores envolvidos na sexualidade humana. A abordagem bioecológica do desenvolvimento humano possibilita analisar a pessoa como um ser ativo e dinâmico, que interage com o tempo e com o contexto que vivencia, modificando e sendo modificada por ele (Bronfenbrenner, 1979/1996).

Não se pode negar a sexualidade dos jovens e associar a sua iniciação apenas a condição psicológica 
da fase em que vivem e a condição socioeconômica que possuem. Os resultados apontam à necessidade de considerar o adolescente dentro dos contextos que formam o seu ambiente ecológico e identificar fatores de risco para que a rede protetiva seja fortalecida e as ações tenham repercussão no desenvolvimento desses.

Aspectos macrossistêmicos e exossistêmicos devem ser considerados, pois interferem diretamente na adesão e no envolvimento dos adolescentes de qualquer atividade. $\mathrm{O}$ conhecimento acerca da idade de iniciação sexual para cada sexo permite organizar ações educativas voltadas para uma vida sexual e reprodutiva saudável. Os programas de controle da fecundidade e prevenção de doenças sexualmente transmissíveis e HIV precisam ser ofertados e acessíveis nos contextos em que estão inseridos. Nesse caso, os serviços de saúde pública não podem simplesmente estar presentes e manter um programa preventivo que iniba o adolescente de participar. A escola é um microssistema que compõe, juntamente com a família, o mesossistema desses jovens. Geralmente, a rede de apoio é escassa e formada por poucos contextos nos quais são mantidas relações estáveis e significativas. Por esse motivo, cabe a escola e a família retomar o cuidado e conduzir o adolescente na identificação de estratégias individuais protetivas para minimizar o risco.

Pelo exposto sobre o assunto e considerando os resultados encontrados no presente estudo, sugere-se que as ações devem estar voltadas para a promoção de bem-estar e devem integrar a pessoa, o processo, o tempo e o contexto como base de reflexão para uma prática protetiva mais efetiva.

\section{REFERÊNCIAS}

Aquino, E. M. L., Heilborn, M. L., Knauth, D., Bozon, M., Almeida, M. C., Araújo, J. et al. (2003). Adolescência e reprodução no Brasil: A heterogeneidade dos perfis sociais. Cadernos de Saúde Pública, 19(2), 377-388.

Borges, A. \& Schor, N. (2005). Trajetórias afetivo-amorosas e perfil reprodutivo de mulheres adolescentes residentes no Município de São Paulo. Revista Brasileira de Saúde Materno Infantil, 5(2), 163-170.

Brandão, E. R. \& Heilborn, M. L. (2006). Sexualidade e gravidez na adolescência entre jovens de camadas médias do Rio de Janeiro, Brasil. Caderno de Saúde Pública, 2(7), 1421-1430.

Bronfenbrenner, U. (1996). A ecologia do desenvolvimento humano: Experimentos naturais e planejados. Porto Alegre: Artes Médicas. (Original publicado em 1979).

Bronfenbrenner, U. \& Morris, P. (1998). The ecology of developmental processes. In W. Damon (Org.), Handbook of child psychology, (pp. 993-1027). New York: Jonh Wiley \& Sons.

Bronfenbrenner, U. \& Evans, G. (2000). Developmental science in the 21st century: emerging questions, theoretical models, research designs and empirical findings. Social Development, 9, 115-125.

Cabral, C. S. (2003). Contracepção e gravidez na adolescência na perspectiva de jovens pais de uma comunidade favelada do Rio de Janeiro. Cadernos de Saúde Pública, 19(2), 283-292.

Canavarro, M. C. \& Pereira, A. I. (2001). Gravidez e maternidade na adolescência: perspectivas teóricas. In M. C. Canavarro (Ed.), Psicologia da gravidez e da maternidade (323-355). Coimbra: Quarteto Editora.

Cowan, P. A , Cowan, P. C. \& Schulz, M. S. (1996) Thinking about risk and resilience in families. In E. M. Hetherington \& E. A. Bleachman (Orgs.), Stress, coping and resiliency in children and families, (pp. 1-38). New Jersey: Lawrence Erlbaum Associates.

Figueiredo, B. (2000). Maternidade na adolescência: consequências e trajectórias desenvolvimentais. Análise Psicológica, 18(4), 485499.

Figueiredo, B. (2001). Maternidade na adolescência: do risco à prevenção. Revista Portuguesa de Psicossomática, 3(2), 221-237.

Falcão, D. V. S. \& Salomão, N. M. R. (2005). O papel dos avós na maternidade adolescente. Estudos de Psicologia (Campinas), 2(22), 205-212.

Figueiró, A. C. (2002). Condições de vida e saúde reprodutiva de adolescentes. Revista Brasileira de Saúde Materno Infantil, 2(3), 291-302.

Gama, S. G. N., Szwarcwald, C. L. \& Lela, M. C. (2002). Experiência de gravidez na adolescência, fatores associados e resultados perinatais entre puérperas de baixa renda. Cadernos de Saúde Pública, 18, 153-61.

Gontijo, D. T \& Medeiros, M. (2004). A gravidez/maternidade e adolescentes em situação de risco social e pessoal: algumas considerações. Revista Eletrônica de Enfermagem, 3(6), Disponível em www.fen.ufg.br

Jacard, J., Dodge, T., \& Dittus, P. (2003). Do adolescents want to avoid pregnancy? Attitudes toward pregnancy as predictors of pregnancy. Journal of Adolescent Health, 33(2), 79-83.

Heilborn, M., Salem, T, Knauth, D, Aquino, E, Bozon, M, Rohden, F., Victora, C., Mccallum, C. \& Brandão, E. (2002). Aproximações socioantropológicas sobre a gravidez na adolescência. Horizontes antropológicos, 8, 3-45.

Leal, I. (2000). Gravidez e maternidade na adolescência. Sexualidade \& Planeamento Familiar, n²7/28, 23-26.

Levandowski, D. C. \& Piccinini, C. A. (2004). Paternidade na adolescência: Aspectos teóricos e empíricos, Revista Brasileira de Desenvolvimento Humano, 14(1), 51-67.

Lima, C. T. B., Feliciano, K. V. O. \& Carvalho, M. F. S. (2004). Percepções e Práticas de Adolescentes Grávidas e de Familiares em Relação à Gestação. Rev. Bras. Saúde Mater. Infant., 4(1). Retirado do www.scielo.br em 12/11/2008.

Manlove, J. (1998). Early motherwood in na intergerational perspective: The experiences of a british cohort. Journal of Marriage and the Family,73, 288-294.

Masten, A. S. \& Garmezy, N. (1985) Risk, vulnerability and protective factors in developmental psychopathology. Em Lahey. B. B.\& Kazdin, A. E. Advances in clinical child psychology 8 (pp.1-52) New York: Plenum Press. 
Ministério da Saúde (2002). Planejamento familiar: Manual para o gestor. Brasília: Ministério da Saúde.

Ministério da Saúde (2006). Marco teórico e referencial da saúde sexual e reprodutiva de adolescentes e jovens. Brasília. Recuperado em 10 de junho de 2008 em www.portal.saude.gov.br

Pantoja, A. L. N. (2003). "Ser alguém na vida": Uma análise sócioantropológica da gravidez/maternidade na adolescência, em Belém do Pará, Brasil. Cadernos de Saúde Pública, 19(2), $335-$ 343.

Pesquisa GRAVAD (2006). Pesquisa de Adolescentes no Brasil. Recuperado em 10 de junho de 2008 em www.portal.saude.gov.br

Reis, A. \& Oliveira-Monteiro, N. (2007). Sexualidade e procriação na ótica de jovens de periferias sociais e urbanas. Revista Brasileira Crescimento Desenvolvimento Humano, 17, 54-63.

Rutter, M. (1985). Resilience in the face of adversity: Protective factors and resistance to Psychiatric Disorder. British Journal of Psychiatry, 147, 598-611.

Rutter, M. (1987). Psychosocial Resilience and Protective Mechanisms, American Journal of Orthopsychiatry, 57, 316-331.

Sabroza, A. R., Leal, M. S., Gama, S. J. N. \& Costa, J. V. (2004). Perfil sócio-demográfico e psicossocial de puérperas adolescentes do município do Rio de Janeiro, Brasil - 1999-2001. Cadernos de Saúde Pública, 20(1), 112-120.

Santos Júnior (1999). Fatores etiológicos relacionados à gravidez na adolescência: Vulnerabilidade à maternidade. Em N. Schor, M. S. Mota, \& V. C. Branco (Org.), Cadernos juventude, saúde e desenvolvimento (pp. 223-229). Brasília: Ministério da Saúde.

Soares, I., Marques, M. C., Martins, C., Figueiredo, B., Jongenelen, I. \& Matos, I. (2002). Gravidez e maternidade na adolescência: Um estudo longitudinal. Em M. C. Canavarro (Ed.), Psicologia da gravidez e da maternidade (pp. 359-407). Coimbra: Quarteto Editora.

Silva, D.V. \& Salomão, N. M. R. (2003). A maternidade na perspectiva de mães adolescentes e avós maternas dos bebês. Estudos de Psicologia, 8, 135-146.

Vieira, L. M., Goldberg, T. B. L., Saes, S. O. \& Dória, A. A. B. (2007). Abortamento na adolescência: um estudo epidemiológico. Ciência \& Saúde Coletiva, 12(5), 1201-1208.

WHO (2004). Adolescent pregnancy: issues in adolescent health and development. WHO Discussion Papers on Adolescence. Geneva: WHO.

Recebido em 24/09/2008

Aceito em 29/10/2009

Endereço para correspondência : Elder Cerqueira-Santos, Departamento de Psicologia. Av. Marecal Rondon, Cidade Universitária Prof. José Aloísio de Campos, Universidade Federal de Sergipe, CEP 49100-000, São Cristóvão-SE, Brasil. E-mail: eldercerqueira@yahoo.com.br. 
